\title{
Structure-property correlation: the Ionic conductivity of selected zirconates
}

\author{
Malitsatsi Jesse Mnguni ${ }^{1}$, Thabang Chiya ${ }^{1}$, Caren Billing ${ }^{1}$, David Billing ${ }^{1}$ \\ ${ }^{1}$ Chemisty, University Of The Witwatersrand, Johannesburg, South Africa \\ E-mail: malitsatsi.mnguni@students.wits.ac.za
}

Binary mixed metal oxides described by the chemical formal A2B2O7, or A2B2O6O' to highlight the uniqueness of one of the oxygen atoms, mostly adopts one of two major structure types, Pyrochlore or Defect Fluorite. Materials with both structures types have been investigated as electrolytes in solid oxide fuel cells (SOFCs) due to their ability to conduct ions and possibly low thermal expansion coefficients. Due to the need improve performance temperatures many investigation into doped pyrochlores have been under taken. Whittle et al. found that adding increasing concentrations of yttrium to a pyrochlore type zirconates makes the structure tend toward the fluorite structure. We report the results from our studies into the structure changes in materials of the form Ln2-xYxZr2O7 (where Ln = La and Sm) using variable temperature PXRD and Rietveld refinements. The ionic conductivity of these solid solutions was also studied as a function of the composition, using electrochemical impedance spectroscopy.

[1] Whittle, K. R. et al. (2008) J. Solid State Chem. 182, 442-450

[2] van Dijk T. and Burggraaf A. J. (1980) Phys. Stat. Sol. 58, 115-125

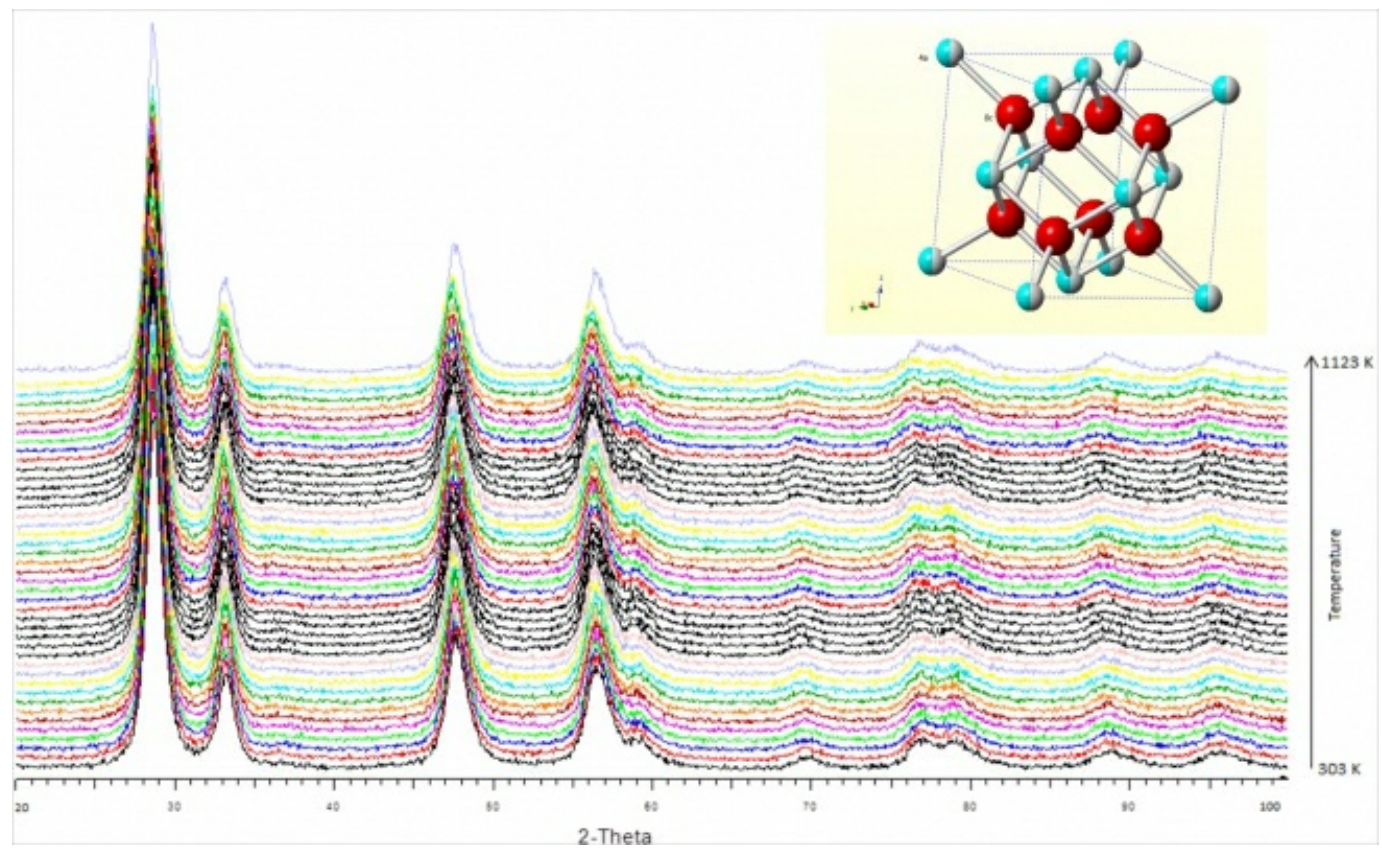

Keywords: Solid oxide fuel cell, Ionic conductivity, Rietveld refinement 